\title{
Diving video-assisted analysis research
}

\author{
Mingquan Long ${ }^{1, \text { a }}$ \\ ${ }^{1}$ Physical Education Department, HuNan Vocational College of commerce, Changsha 410205, \\ Hunan, China \\ aemail: matlab_wjf@126.com
}

Keywords: diving sports, motion simulation, model tracking , video analysis, auxiliary training.

\begin{abstract}
According to the characteristics of the diving sports, a video analysis system is developed for diving sports in the paper. This system is mainly used in assisting coach to instruct athletes more effectively, and it can help the judgers give the correct judgments. Three components are composed in the research, the video analysis, 3D motion simulation and edit interface and the management of database. The background and meaning of developing this system is stated in the first section, and then the relation and the data flow are analyzed among the main modules. In the end, the key techniques and innovations are addressed in detail. In practice, this system can not only improve the efficiency of training, reduce the repeated training and the probability of false judgments, but also there is the high efficiency in the system and it is easy to be operated.
\end{abstract}

\section{Introduction}

All along, the level of China's diving are a world leader, mainly because we have an experienced coaching staff and the talented and hard training athletes. However, in recent years, the dominance of the diving project is seriously challenged, like Canada, the U.S. and Australia the rising star, the gold target of diving sport athletes had a huge impact at all leve of diving competitionls. The main reason for this phenomenon is because of our research is still relatively backward sports, science and technology in diving training is almost zero. The United States, Australia and other countries produces sharp contrast to China diving training, the computer-aided motion analysis system CAMAS (Computer Assistant Motion Analysis System) are using advanced without exception. CAMAS is the product of a variety of IT integration, which includes the multi-disciplinary field of image processing and analysis, computer vision, pattern recognition, artificial intelligence, computer graphics, mathematics, kinesiology and other knowledge and technology. Three-dimensional simulation exercise training and video analysis system is with real-time and the full range of observation, the merits of a training or competition can be quantified in the form of analysis and evaluation, so CAMAS play an increasingly important role.

Rapid progress has been made for sports video analysis system in recent years, related hardware and software system has been initially applied to a variety of sports training. Kanav Kahol had layered the behavioral segmentation method, dance gesture is segmented from the motion sequences of dance, dance training is identifed and guidanced[1]. In order to improve the competitive level of our athletes, promote the development of China's sports undertakings, Institute of Computing Technology had developed simulation of three-dimensional human motion video analysis system for sports training, where there are the digitized three-dimensional human motion in computer simulation technology, human Sports Biomechanics data with real human motion data, there are graphically realistic three-dimensional simulation, design, analysis of technical movements, and there is highly training guidance capability in competitive sports [2,3]. However, the system also needs to be further enhanced and improved in order to achieve a higher degree of utility. For example, in diving, and for an action, there are a series of the quality of athletes completing action and athletes, athletes weight, height, the proportion of the various parts of the body, take-off angle, each node acceleration, flipped operation timing in the air, the show arm timing before entering the water, and angles into the water, these problems still be not completely solved, what is the relationship between these data, and what factors it is related to, what kind of 
law is existed between them, these are pending further study. Therefore, a diving video motion analysis system is to be designed and developed, it is of great help for improving the competitive level of diving athletes, and it is a very important significance.

\section{Diving motion video analysis system framework}

Diving motion video analysis system consists of three modules: a video analysis module, 3D motion simulation and graphical editing interface module, and database management module. The main process is shown in Figure 1.

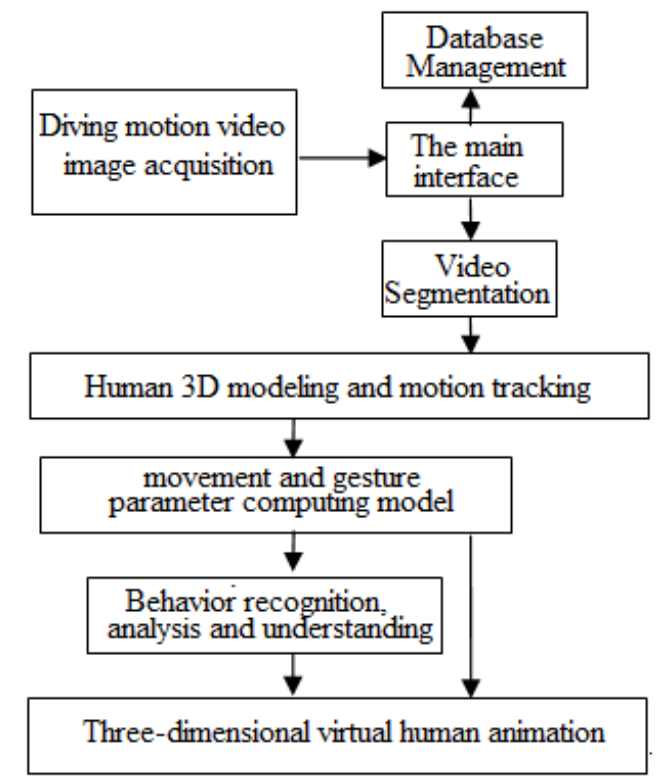

Fig.1. Diving sport video analysis system process

First, the existing diving video or real-time training video are imported the main interface (Figure 2), which is captured from the camera, this video is split. Motion tracking is done based on segmentation. The purpose of tracking is to obtain motion parameters of each athlete's joints, mathematical modeling of these parameters are established by using regression analysis, the model is built with athlete motion parameters and attitude parameters.

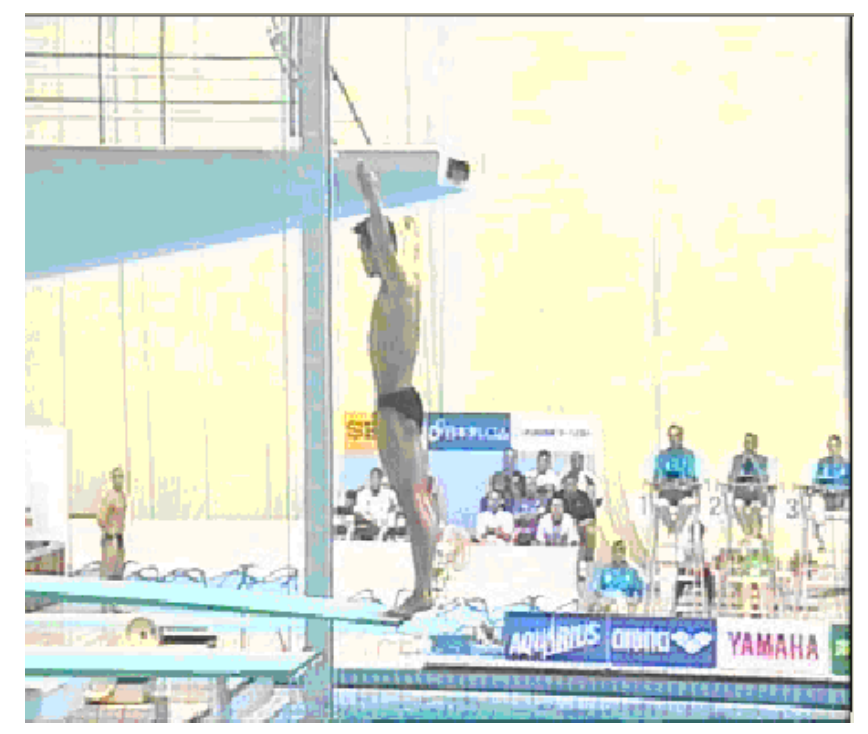

Fig.2. Main interface

A set of motion parameters are obtained by tracking, and the 3D virtual human is drived, athletes training is simulated by a virtual human for the whole process, so that coaches, athletes and referees can observe the completed situation of the dive from a comprehensive, multi-angle. In addition, with such a 3D graphics editor interface, the action can be also modified, the basic 
movements are designed, operation is integrated, and the actual situation is contrast to virtual one.

The athlete data management and user management are mainly completed in Database management. For an athlete, some of his basic information, such as height, weight, movement difficulty coefficient, play an important role the results of the training judgement, in general, these data are relatively fixed, so these can be save in the database, artificial troubles should be reduced in each training set. In order to avoid abuse and misuse of system functions, User management is defined as that users are created by the super administrator, and different users are given with different permissions.

\section{Video analysis technology}

Video Segmentation: When diving video is shooted, in order to capture the full track athletes, the camera movement is generally made. This background motion,which caused by the camera motion, is called global movement [4,5]. Typically, there is a global motion in diving video, so to obtain accurate global motion parameters are that human key and basic of athletes are extracted from the video.

Taking into account the characteristics of diving video, we use the three-parameter camera motion model [6], such as the formula (1).

$$
\left[\begin{array}{l}
v_{x} \\
v_{y}
\end{array}\right]=a\left[\begin{array}{l}
x \\
y
\end{array}\right]+\left[\begin{array}{l}
a_{x} \\
a_{y}
\end{array}\right]
$$

Where, $a=z_{x y}, a_{x}=f_{x}\left(p_{x}, z_{x y}\right), a_{y}=f_{y}\left(p_{y}, z_{x y}\right) . \quad z_{x y}$ is a camera zoom (scaling) factor, and $\left(p_{x}, p_{y}\right)$ is a translation components in the $\mathrm{x}$ and $\mathrm{y}$ axis of the camera panning.

According to the human skin model, the current frame $I_{t}$ and the previous frame $I_{t-1}$ is respectively pretreated by operation, the athletes are extracted from the current frames $I_{t}$ and the previous frame $I_{t-1}$. Thereafter, the closing operation is made for the current frame $I_{t}$ image and the previous frame $\mathrm{I}_{\mathrm{t}-1}$ image, the communicated image is obtained between a current frame $\mathrm{I}_{\mathrm{t}}$ and a previous frame $\mathrm{I}_{\mathrm{t}-1}$. Then, at the exclusion of the majority of the foreground image, it is macro-block match. In order to improve the matching speed of macroblock, by KMP string pattern matching algorithm [7] revelation, we designed a no backtracking image block matching algorithm (NBT).

Model Tracking:Video segmentation is for tracking service, the motion parameters of each athlete's joints can be g0tten from the original video by tracking. Tracking methods, which is currently used, are block-matching method (Block Matching), optical flow method (Optical Flow). In this paper, feature point tracking method is used and it is based on optical flow method.

Calculating Model:Motion parameters and attitude parameters are important data to analyze gymnastics, diving, it is used to describe the state of motion of athletes. The motion parameters of various joints are instantaneous velocity, acceleration, etc., the pose parameters are mainly height and direction angle in each part of the body, and the geometric relationships between them. Calculation model is estblished based on the extracted joint motion parameters and body posture parameters, it is for the calculation of motion parameters and pose parameters at any time point in each joint. To calculate motion parameters and pose parameters at any time or anywhere, the physiological characteristics of human and biological movement principle are studied, the way and the limits are to understood in the movement changes of the human body various parts, on this basis, human motion tracking model is combined, a computational model and calculation methods are researchd about in the diving joint points and body parts motion parameters, pose parameters.

Behavior recognition and understanding:The motion trajectory, the motion parameters and attitude parameters, which have been obtained, is used as a basis for recognition analysis. Athletes 
actions identify is also semantic classification category. The dive is decomposed into 13 kinds of basic actions class, which are the run, arm stand in off stage, swivel, forward, reflexive, backward and inward somersault, straight body air stages, curve body, tuck, somersault and twist, into the water, and then they are combined into a dive actions, such as 207B backward flipped curved body with three weeks and a half, the machine is enabled to automatically identify diving video, the identification result is used for real-time operation of automatic annotation, and it is action base on search criteria.

Motion Analysis ultimate goal is to be able to analyze the deficiencies of athletes actions to guide athletes training. Athletes actions are compared with standard actions in database to get numerical differences, then after adjusting the semantic mapping process, the pre-defined rules are converted in the form of natural language to describe the completion of the action, it includes the overall level evaluation of completing action, it is decomposed into four parts, which are the run-up, takeoff, air, into the water, these basic movements are essentials to grasp the situation, where should be improved and how to improve the place.

\section{Conclusion}

The system provides a rich set of functions, some of the potential demand is constantly digging. From the trial process, the use of the system can help players master the technical essentials of action as soon as possible, training efficiency is greatly improved and duplication of training blind is reduced. The possibility of injury athletes is reduced.

With this training video analysis and human diving simulation system, diving training mode changes from traditional training to science one, diving training changes from the coache eye method and athletes repeated training to the precision video capture, human motion analysis, and simulation. In addition to diving motion video analysis system is used to training, there is a great value. For example, during the diving competition, it can assist the referee to judge the diving competition, to avoid false positives which is caused by subjective factors, objectivity, fairness and impartiality of referees work are improved.

Diving sports video analysis system is integration of computer graphics, digital image processing, pattern recognition, biology, mathematics and human motion technology, the system is used as a specialized diving support system, which departure from diving video and diving itself features, they will have a significant impact on the diving training. In the future, the diving team will try and feedback through the system, and its capabilities are constantly improved, it can be used officially for training and competition.

\section{References}

[1] Kanav Kahol, Priyamvada Tripathi, Sethuraman Panchanathan. Automated gesture segmentation from dance sequences[C]. Proceedings of the Sixth IEEE International Conference on Automatic Face and Gesture Recognition, 2004.

[2] Tao Kun, Wu Si, Lin Shouxun, etc. Research on Panorama Composition Technique of Sports Video[J], JOURNAL OF COMPUTER-AIDED DESIGN \& COMPUTER GRAPHICS, 2005, 17(11)

[3] Wang Zhaoqi, Zhang Yongdong,Xia Shihong.

3D Human Motion Simulation and a Video Analysis System for Sports Training[J], JOURNAL OF COMPUTER RESEARCH AND DEVELOPMENT, 2005, 42(2)

[4] D. Farin, P. H. N. de With, and W. Effelsberg, Video-object segmentation using multi-sprite background subtraction[C], in Proc. IEEE International Conference on Multimedia and Expo (ICME), 2004.

[5] Yaakov Tsaig, Amir Averbuch. Automatic Segmentation of Moving Objects in Video Sequences :A Region Labeling Approach[J]. IEEE Transactions on Circuits and System for Video Technology, 2002, 12(7). 
[6] WANG Jia,WANG Hai-Feng,,LIU Qing-Shan, etc. Fast Global Motion Estimation Based on 3-Parameter Global Motion Model[J], CHINESE JOURNAL OF COMPUTERS, 2006, 29(6)

[7] Thoms H. Cormen,Charles E.Leiserson,Ronald L.Rivest. Introduction to algorithms[M]. The MIT Press,2001.

[8] Wang Chengyu, Gu Guanghua. Video object segmentation and tracking algorithm based on difference and intersection[J], OPTICAL TECHNIQUE, 2004, 30(5) 